\title{
Combined approach for NSCLC with chest wall invasion: the role of VATS
}

\author{
Jury Brandolini ${ }^{1}$, Giampiero Dolci ${ }^{1}$, Filippo Antonacci ${ }^{1}$, Niccolò Daddi ${ }^{1}$, Pietro Bertoglio ${ }^{1}$, \\ Kenji Kawamukai ${ }^{1}$, Barbara Bonfanti ${ }^{1}$, Francesca Calabrese ${ }^{1}$, Sergio Fortiparri ${ }^{1}$, Alessandro Gasbarrini ${ }^{2}$, \\ Piergiorgio Solli ${ }^{1}$
}

${ }^{1}$ Division of Thoracic Surgery, Azienda Ospedaliero-Universitaria di Bologna, via Albertoni 15 Bologna, Italy; ${ }^{2}$ Department of Oncologic and Degenerative Spine Surgery, IRCCS Istituto Ortopedico Rizzoli, Bologna, Italy

Contributions: (I) Conception and design: J Brandolini, P Solli; (II) Administrative support: J Brandolini, P Solli; (III) Provision of study materials or patients: J Brandolini, P Solli; (IV) Collection and assembly of data: J Brandolini, P Solli; (V) Data analysis and interpretation: J Brandolini, P Solli; (VI) Manuscript writing: All Authors; (VII) Final approval of manuscript: All Authors.

Correspondence to: Jury Brandolini, MD. Division of Thoracic Surgery, Azienda Ospedaliero-Universitaria di Bologna, via Albertoni 15 Bologna, Italy. Email: jury.brandolini@gmail.com; jury.brandolini@ausl.bologna.it.

\begin{abstract}
To date the conventional open surgery has been the standard surgical management of nonsmall cell lung cancer with chest wall invasion, which is seen in less than $10 \%$ of patients with lung cancer in mostly reported series. Minimally invasive techniques have emerged as alternative approaches for the treatment of locally advanced and more complex cases, with a demonstrated oncologic outcomes equivalent to open thoracotomy and lower overall complication and mortality rates. Despite, there are few published studies, the en-bloc chest wall resection with vertebrectomy can be perform by a video-thoracoscopic hybrid techniques in selected patients with lung cancer invading the spine. In this article, we describe our surgical approach and technical aspects of VATS hybrid approach for T4 vertebra combined resection. In addition, the different minimally invasive approaches described in the existing literature are briefly examined.
\end{abstract}

Keywords: Lung cancer; vertebrectomy; video-assisted thoracoscopic surgery (VATS); chest wall; minimally invasive surgery

Received: 30 May 2020; Accepted: 21 August 2020; Published: 20 July 2021.

doi: 10.21037 /jovs-20-138

View this article at: http://dx.doi.org/10.21037/jovs-20-138

\section{Introduction}

The prevalence of non-small cell lung cancer (NSCLC) with chest wall invasion is less than $10 \%$ (from $5 \%$ to $8 \%)$ in mostly reported series (1). The complete surgical resection via lobectomy with en-bloc chest wall resection represents the gold standard for curative intent, with a 40 $50 \%$ expected 5 -years survival when a complete resection is achieved (R0) and when there is no nodal involvement (N0).

The adjunct of chest wall resection and reconstruction carries a not negligible increase in terms of morbidity and mortality (up to $9 \%$ in recent series) if compared to conventional standard lung resections (2).

Historically, the first chest wall resection in continuity with a lung cancer was described by Coleman in 1947, who performed a pneumonectomy with en-bloc resection of rib cage (3), but for long time, despite sporadic reports, NSCLC invading the chest wall ( $\mathrm{T} 3$ tumour according to the $8^{\mathrm{Th}} \mathrm{TNM}$ staging system) (4) has been considered a contraindication for upfront surgery, mostly due to technical reasons.

What was once anticipated as a notable surgical challenge, is no longer a technical problem and en-bloc chest wall and lung resections are today routinely performed with excellent outcomes and thanks to a multitude of techniques and materials available for the step of the reconstruction.

The modern issue nowadays is rather represented by the clever and integrated use of the other non-surgical modalities (chemo- and/or radio-therapy), with the ultimate 


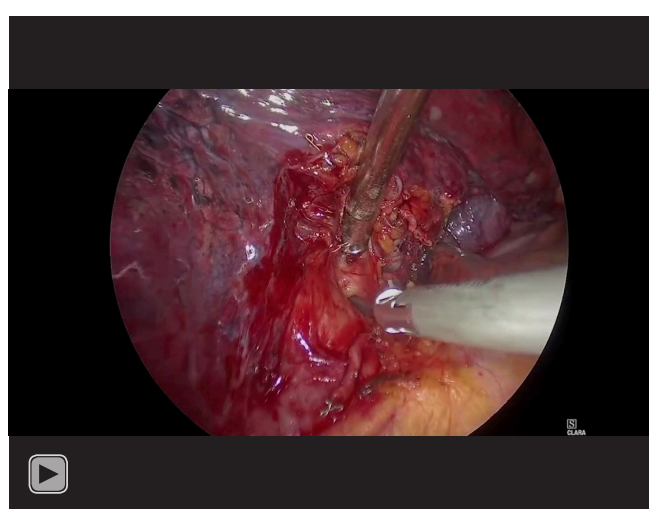

Video 1 Bi-portal video-assisted thoracoscopic lobectomy with enbloc chest wall resection and T3/T4 hemi-vertebrectomy.

goal of increasing the chance of a complete resection, of a better control of lymphatic/haematogenous spread, and eventually to enhance the overall chance of long term survival.

The tumours arising in the superior sulcus (i.e., Pancoast tumours) that occur in $1 \%$ of patients with NSCLC are a complete separate chapter and for their specific peculiarities will not be included in the present discussion. For the same reason the topic of the different options available in terms of reconstruction will not be covered in this article.

Due to the rapidly mounting evidence of safety and efficacy of minimally invasive approaches for the treatment of NSCLC, with proved oncologic outcomes equivalent to open thoracotomy and lower overall complication and mortality rates (5-7), there has been a prominent interest on the use of minimally invasive approaches also for the treatment of locally advanced and more complex cases (chest wall and/or lymph node involvement, bigger lesions, previous chemo- or radio-therapy, vascular and bronchial sleeve resections).

The role and efficacy of VATS for these more complex procedures, specifically in the context of videothoracoscopic hybrid techniques for lobectomy with chest wall resection/reconstruction, should be considered still under investigation as there are few published studies, though feasibility and safety have repeatedly been reported by different Authors (8-12).

In this article, we will review some of the aspects of the available technical options for the cure of locally advanced T3 NSCLC that infiltrates the chest wall approached via VATS and present a case report (Video 1) of VATS hybrid approach for $\mathrm{T} 3 / \mathrm{T} 4$ vertebra combined resection.

\section{Surgical technique}

Pre-operative staging work-up includes a whole-body computed tomography scan (CT scan), a 18-FDG positron emission tomography-computed tomography (PET-CT) and the brain imaging (via CT/MRI) as per routine for all patients with suspected or proven lung cancer. Cardiopulmonary tests to assess the functional reserve are also done as per routine in any lung resection.

In case of chest wall invasion, occasionally an additional MRI is performed in case of tumours arising close to the vertebrae in order to better assess the involvement of the column or foramina.

In summary three main approaches have been described; all these options have been proved valuable, they present advantages and disadvantages and the ultimate choice of the best approach is eventually related with the specific features of the clinical case, with the surgeon skill and attitude and eventually to the locally available resources.

\section{Chest wall resection first/no reconstruction}

Widmann et al. in 2000 described the first complete VATS left upper wedge resection en-bloc with chest wall (two ribs) without reconstruction of the defect, in a patient treated by preoperative radiotherapy for a T3 chest wall lung adenocarcinoma (12). Demmy et al. reported a minimally invasive lung and rib resection via a multiple-port VATS approach; the area of chest wall invasion is approached first, with standard long ribs cutter introduced through a $4-\mathrm{cm}$ utility incision. Once the chest wall portion is resected is left in place attached to the lobe and the subsequent VATS lobectomy is achieved in a standard thoracoscopic manner, depending on surgeon's experience. With this technique, small areas of infiltration, preferably posteriorly located, well covered by muscles and scapula are the ideal condition as the reconstruction of the wall defect is normally not required (13).

\section{VATS lobectomy first/chest wall resection via counter- incision/reconstruction}

The "hybrid thoracoscopic approach" described by Berry et al. from Duke University, envisages a standard video-assisted thoracoscopic lobectomy with radical lymphadenectomy followed by chest wall resection performed through a small counter-incision made precisely above the area of chest wall invasion; thus the exact ribs 

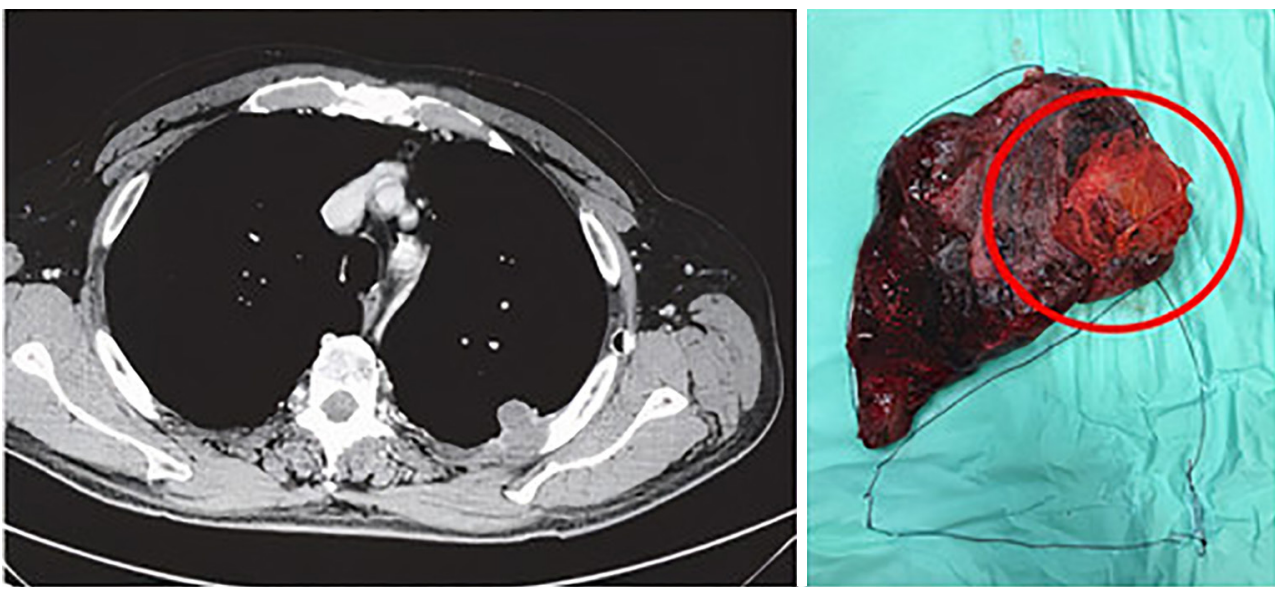

Figure 1 Preoperative CT-scan and the surgical specimen of a pT3N0M0 squamous cell carcinoma invading the 4th rib approached via VATS hybrid technique (courtesy P Solli J Vis Surg 2017;3:73).

to be resected are directly visualized and a potential reconstruction with prosthetics materials can be done thoracoscopically or through the space of the counterincision. The specimen is removed through the posterior counterincision (8). A variation of this approach, sharing the same philosophy, is the similar one described by GonzalezRiva where the lung resection is performed first through a single anterior utility-incision (uniportal VATS) and is then associated with a posterior counter-incision for ribs transaction and with the reconstruction step if required (14). This is also our preferred technique (with 2 ports approach) for patient with limited T3 chest wall invasion $(15,16)$ (Figure 1) and has been reported by other Authors in the recent literature (17).

\section{Chest wall resection under VATS guidance first/lobectomy via chest wall defect}

An additional option could be considered the original technique described by Bayarri; this includes an alternative hybrid approach where the area of chest wall invasion is initially confirmed and accurately assessed from inside the chest through a single-port VATS incision; a limited counter-incision is then made in correspondence of the borders of infiltrated chest wall and eventually the ribs are precisely transected under direct vision.

Lung resection with radical nodal dissection is finally performed via the chest wall defect. Among advantages advocated by the Authors are (I) an additional intraoperative staging (pleura, lymph nodes); (II) no rib spreading with reduced surgical trauma and precise visualization of the chest wall infiltration; (III) no need of large experience in VATS lung resection; (IV) accurate chest wall resection boundaries (18).

\section{VATS lobectomy}

The operation is performed under general anaesthesia with double-lumen intubation and on one-lung ventilation; the patient is placed in the lateral decubitus position and prepped with a careful exposure of the area of expected chest wall invasion. Even though the procedure is scheduled to be fully undertaken via minimally invasive approach, the whole team is prepared for conversion to an open approach if needed at anytime and the surgical instrumentation for thoracotomy, chest wall resection and reconstruction is available in the theatre room from the beginning. No differences are associated with the use of uni- or multi-port VATS technique and the final decision is eventually related to surgeon's preference.

Our standard among different approaches for VATS lobectomy is for a bi-portal technique, with a $3-4 \mathrm{~cm}$ utility incision in the $4^{\text {th }}$ or $5^{\text {th }}$ intercostals (depending on lobes) space on the anterior axillary line and an additional $10-\mathrm{mm}$ port in the mid-axillary line for the $30^{\circ}$ thoracoscope. After a preliminary careful inspection of the entire chest cavity in order to exclude the presence of accidental metastatic deposits on the pleura, the area of chest wall invasion is cautiously visualised and a plan of the extent of ribs resection is established. The advantage of having two chest access allows the thoracoscope to be advanced alternatively through the $10-\mathrm{mm}$ port or the anterior utility-incision, in order to maximise the visualization. 
The VATS lobectomy is then carried out according to any standard thoracoscopic technique for lung resections: hilar dissection, individual ligature and division of pulmonary artery branches, pulmonary vein and lobar bronchus; fissure division according to the fissure-less technique; hilar and mediastinal nodal dissection.

Maximal attention should be paid during the lung resection in order to avoid excessive lung parenchyma traction and mobilization thus stretching the area of chest wall infiltration with consequent disruption and spillage of tumour within the pleural cavity (no-grasping technique).

Occasionally the level of ribs infiltration might preclude an expedite and accurate lymph nodes dissection because the lung cannot be adequately mobilized. In this case it is advisable to complete first the lung and en-bloc chest wall procedure and postpone the lymphadenectomy at the end after retrieval of the surgical specimen.

\section{Chest wall resection/reconstruction}

At this stage, depending on the degree and topography of chest wall invasion different options are available.

The advantage of the VATS availability relies on the chance to have direct guidance of the high-definition camera and an easier condition to obtain adequate free margins.

Chest wall resection might be accomplished through a guided and precise counter-incision directly over the involved area, without scapular mobilization or ribs spreading; the soft tissue with intercostals neurovascular bundles might be divided using cautery and energy device scalpel also through the working port.

Ribs transection can be also performed using longhandled rib shears or endoscopic osteotomes moved directly through the utility-incision depending the favourable angle with the bones.

Other options include the high-speed drill burr and the Gigli saw again guided from the inside cavity view.

The specimen retrieval is a very delicate phase because of the size of lobe with the attached rib/ribs. A wound protector and a robust extraction sac are both necessary for a safe specimen extraction, that can achieve both through the working port or through the counter-incision.

In order to avoid bag perforation and tumour spillage, the endoscopic instruments should be used to perpendicularly orient the lobe in line with the exit point and then extracted through the utility port.

If needed the skin incision must be enlarged (both of the utility port or the counter-incision, depending on the preferred route of extraction). Occasionally an additionally incision could be considered for an easier specimen retrieval in the sub-xiphoid space but this has never been needed in our experience.

The indication for chest wall reconstruction follows the same rules as per classic open thoracotomic approach for lung cancer, the primary goals being (I) to maintain the pulmonary mechanic, (II) to restore chest wall rigidity for intrathoracic organs protection and (III) to prevent lung herniation and paradoxical movements.

As a results small apico-posterior located defects if well covered by the scapula and muscles might not require any reconstruction. On the contrary, it may be required in case of anterior chest wall resection, if the defects is large (over the $5 \mathrm{~cm}$ in diameter) and for the for defects involving two or more ribs, in order to restore a good chest wall stability and strength, and to prevent any kind of chest flail or scapular tip entrapment.

With the increased availability of reconstruction materials, currently a multitude of synthetic, biologic, and metallic materials are available to reconstruct any type of chest wall defects $(19,20)$.

\section{Case report (video)}

A 57-year-old smoker female was admitted to our department presenting with a 4-weeks history of persistent right-sided chest and shoulder pain and a round opacity in her right upper lobe on chest $\mathrm{X}$-ray.

The clinical preoperative staging included a total body CT-scan, a PET/CT scan and a MRI of the brain and of the thoracic column. A $4 \mathrm{~cm}$ mass in the right upper lobe was discovered with invasion of the posterior arch of the 3 th and $4^{\text {th }}$ rib also abutting the pleural surface; on MRI the right lateral portion of the $\mathrm{D} 4$ vertebral body proved involved with associated infiltration of the corresponding transverse process and D4-D5 vertebral foramen invasion, but without spinal canal invasion. A CT-scan guided fine needle biopsy revealed a lung adenocarcinoma (TTF1pos, EGFR wt, ALK neg, ROS-1 neg, PD-L1 10\%). An extensive mediastinal staging with EBUS was undertaken to rule out unforeseen mediastinal N2-disease and the final clinical stage resulted to be cT4N0M0.

After tumor board discussion, a multimodality treatment was decided and the patient started on an induction neoadjuvant chemotherapy regimen with Gemcitabine + Cysplatin according to standard dosage for 4 cycles. The treatment was well tolerated and the post-induction 

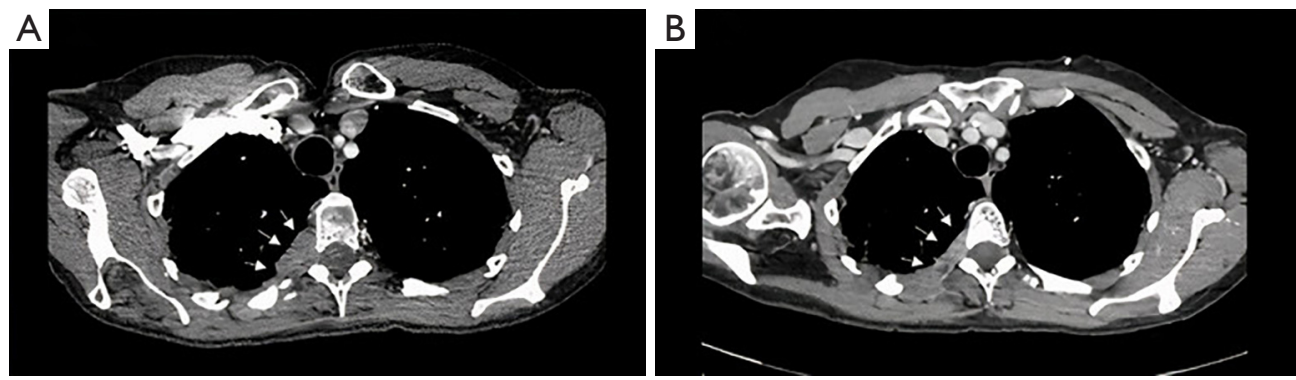

Figure 2 Chest CT-scan before (A) and after (B) induction chemotherapy showing a right upper lobe adenocarcinoma invading the posterior aspect of the $3 \mathrm{rd}$ rib and the lateral aspect of vertebral body.

restaging images (CT, PET/CT and MRI) demonstrated a major radiological response in the right upper lobe with only residual disease at the level of $\mathrm{D} 4$ posterior costotransverse angle with adjacent marginal vertebral invasion (Figure 2).

The case was then re-assessed during the multidisciplinary team board (MDT) and an orthopaedic surgeon with specific experience in oncology and surgery of the spine was involved.

The pulmonary, cardiac and anaesthesiology evaluation were in favour of surgery and then on the basis of the favourable response, the excision was indicated.

\section{Step 1. RUL VATS lobectomy}

A bi-portal VATS technique was performed, with an anterior to posterior dissection of all the hilar structures.

The entire pleural surface was inspected to verify the absence of metastasis; the dorsal segment of the right upper lobe proved to be attached to the chest wall. This area of infiltration was carefully inspected and the vertebral body involvement was intra-operatively confirmed.

The right upper lobectomy was undertaken in a standard fashion.

The lung was gently retracted posteriorly and the anterior mediastinal pleura opened along the anterior hilum with exposure, dissection and isolation of the superior pulmonary vein and the anterior trunk of the pulmonary artery (Boyden trunk) (35 mm powered vascular stapler). The intermediate pulmonary artery was then followed upon the take-off of the postero-ascending pulmonary A2 artery branch, divided with a $35 \mathrm{~mm}$ powered vascular stapler.

Once the artery is divided, the removal of lymphatic tissue helps in the identification of the carina between the upper and intermediate bronchus; the lobe is now pulled upward to expose the upper bronchus that arises vertically that after isolation it is divided with a $60-\mathrm{mm}$ green powered stapler. All the staplers for vascular and bronchus division are introduced and advanced from the thoracoscopic port to more easily tackling the hilum.

The horizontal fissure and the posterior part of the oblique fissure are divided with a $60-\mathrm{mm}$ green powered stapler and the upper lobectomy completed with the "fissure-last" technique. With the lobe left in place and still attached to D4 a radical node dissection is performed (station 2R-4R-11R-7).

The chest wall resection is left as second step but is commenced from inside via the VATS procedure; the borders of involved area are encircled precisely through the incision of the parietal pleura with a monopolar electrocautery scalpel and with exposure of the periostium on the inner face of the ribs; with the energy device (Ethicon Harmonic ${ }^{\circledR}$ Scalpel)the intercostals muscles are divided; the III-IV and V ribs are sectioned with an endoscopic osteotome introduced through the working port; free macroscopic margins are precisely delimited in order to achieve radical margins and completely release the tumour. A single $28 \mathrm{~F}$ is left in place, the residual lung ventilated, the bronchial stump and stapling line checked for air-leak and the chest utility incision closed in layers.

\section{Step 2. chest wall resection (Hemivertebrectomy)}

Patient is placed in prone position and a posterior midline incision is performed (from T2 to T8).

The amount of vertebra invasion and a plan for extension of the resection is accurately evaluated on preoperative MRI. Briefly the posterior procedure includes insertion of pedicle screws, removal of the laminae, uniateral costotranversectomy resection of the nerve roots, and release of the dura from the tumor, section of the vertebral 

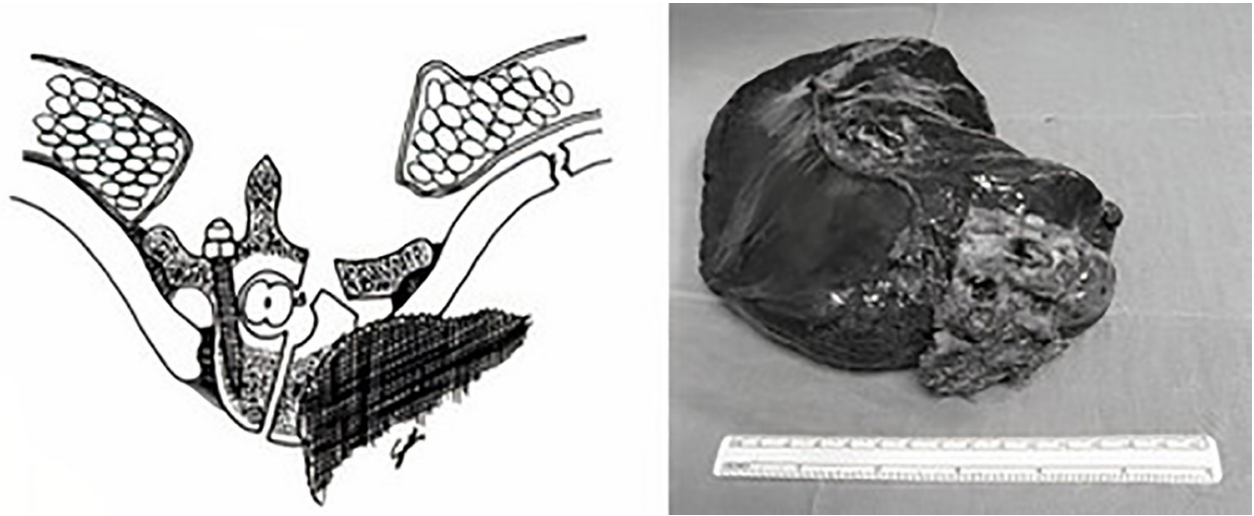

Figure 3 Surgical specimen showing the right upper lobe en bloc with the T3/T4 hemi-vertebrectomy with a drawing summarising the technique for vertebral resection.
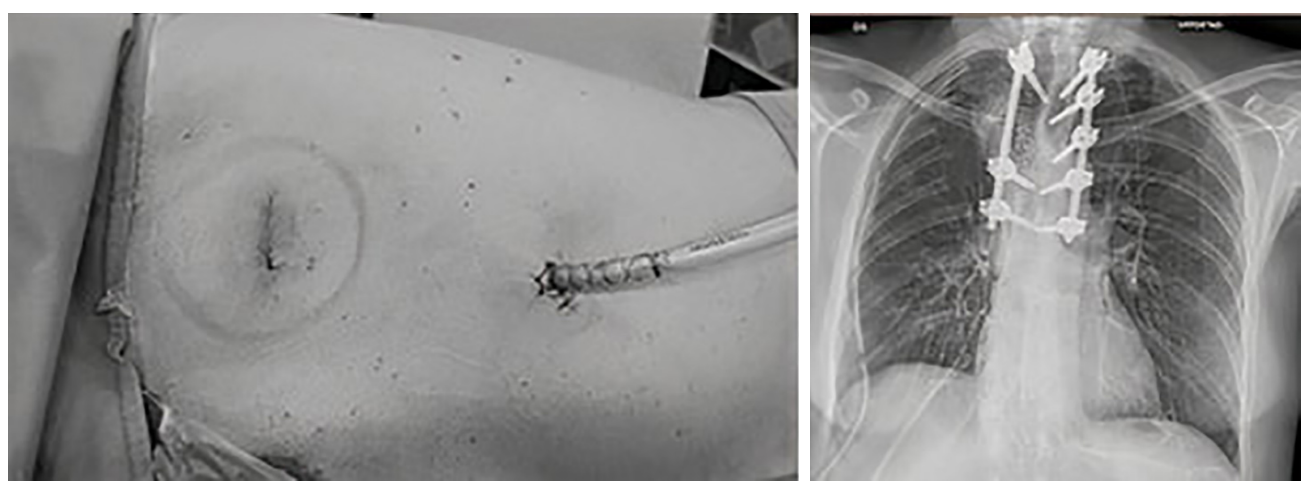

Figure 4 The bi-portal VATS approach utilized for lobectomy and the postoperative chest X-ray showing the spine stabilization with titanium bars implant.

body. The first stage is a right laminectomy at the level of the lesion extended one level above and one level below; the nerve roots and accompanying vessels are identified and divided within the spinal canal in order to prevent cerebrospinal fluid leakage. The caudal and cephalad discs were then removed. The posterior vertebral osteotomy of T3 and T4 was done using ultrasonic scalpel and osteotomes while maintaining anterior control and protection of the major surrounding organs with malleable retractors. The specimen is carefully removed en-bloc with the right upper lobe (Figure 3).

The posterior stabilization was accomplished with titanium bars (Ennovate ${ }^{\circledR}$ B Braun system - MRI compatible) from T2 to T6 (Figure 4). The reconstruction of the ribs was deemed not necessary because of the posterior site of the chest wall defect, under the scapula and $<5$-cm in diameter.
The entire procedure was performed by a dedicated team of orthopaedic surgeons.

Post-operative course was uneventful; the chest drain was removed on day 5 and the patients was discharged home the on day $7^{\text {th }}$. The final histology showed an ypT4 N0 M0, TNM $8^{\text {th }}$ radically resected (R0). MDT final decision was in favour of radiological follow up with no need of adjuvant therapies.

After 6 months' follow-up patient is well with a good performance status and whole-body CT scan showed no evidence of local or distant relapse.

\section{Key concepts}

(I) The VATS hybrid approach with en-bloc resection of chest wall with the involved lung, is able to minimize the surgical trauma and provides a safe and 
viable option in selected case of lung cancers with limited chest wall invasion;

(II) The presence of chest wall infiltration does not preclude the use of minimally invasive technique, being the two steps (lung resection and chest wall resection +/- reconstruction) feasible separately with different techniques (see text for details);

(III) carefully selection of cases (limited and posterior area of infiltration) and the usage of modern technology (UHD 4K camera, energy-based tissue sealing device) are both crucial to facilitate the procedure and to achieve good outcomes.

\section{Conclusions}

To date, only small series and few retrospective studies in literature report the application of video-assisted thoracoscopic surgery in the treatment of NSCLC invading the chest wall or the spine; there are several reason for this limited utilisation: low incidence (5-8\%), demanding cases, debated advantage in terms of pain control and oncological safety, need of elevated technical skill in VATS approach.

Nevertheless, minimally invasive technique may offer several short-term advantages in term of reduced length of stay, low postoperative pain (reduced consumption of analgesic drugs), better preservation of pulmonary function especially in elderly and frail patients, improved quality of life and recovery to normal life.

The area of chest wall invasion is in adjunct precisely visualized during VATS and the opportunity to exactly tailoring the extent of chest wall resection without compromising radicality of the surgery is enhanced.

\section{Acknowledgments}

Funding: None.

\section{Footnote}

Provenance and Peer Review: This article was commissioned by the Guest Editor (Federico Rea) for the series "Alternative Surgical Approaches for Challenging Cases in Thoracic Surgery" published in Fournal of Visualized Surgery. The article has undergone external peer review.

Conflicts of Interest: All authors have completed the ICMJE uniform disclosure form (available at https:// jovs.amegroups.com/article/view/10.21037/jovs-20-138/ coif). The series "Alternative Surgical Approaches for Challenging Cases in Thoracic Surgery" was commissioned by the editorial office without any funding or sponsorship. The authors have no other conflicts of interest to declare.

Ethical Statement: The authors are accountable for all aspects of the work in ensuring that questions related to the accuracy or integrity of any part of the work are appropriately investigated and resolved. All procedures performed in this study were in accordance with the Helsinki Declaration (as revised in 2013). The manuscript is waived from patient informed consent according to the ethics committee or institutional review board.

Open Access Statement: This is an Open Access article distributed in accordance with the Creative Commons Attribution-NonCommercial-NoDerivs 4.0 International License (CC BY-NC-ND 4.0), which permits the noncommercial replication and distribution of the article with the strict proviso that no changes or edits are made and the original work is properly cited (including links to both the formal publication through the relevant DOI and the license). See: https://creativecommons.org/licenses/by-nc-nd/4.0/.

\section{References}

1. Goldstraw P, Crowley J, Chansky K, et al. The IASLC Lung Cancer Staging Project: proposals for the revision of the TNM stage groupings in the forthcoming (seventh) edition of the TNM Classification of malignant tumours. J Thorac Oncol 2007;2:706-14.

2. Ettinger DS, Akerley W, Bepler G et al. Non-small cell lung cancer. J Natl Compr Canc Netw 2010;8:740-801.

3. Coleman FP. Primary carcinoma of the lung, with invasion of the ribs: pneumonectomy and simultaneous block resection of the chest wall. Ann Surg 1947;126:156-68.

4. Goldstraw P, Chansky K, Crowley J et al. The IASLC Lung Cancer Staging Project: Proposals for Revision of the TNM Stage Groupings in the Forthcoming (8th) Edition of the TNM Classification for Lung Cancer. J Thorac Oncol 2016;11:39-51.

5. Riquet M, Arame A, Le Pimpec Barthes F. Non-Small Cell Lung Cancer Invading the Chest Wall. Thorac Surg Clin 2010;20:519-27.

6. Lanuti M. Surgical Management of Lung Cancer Involving the Chest Wall. Thorac Surg Clin 2017;27:195-9.

7. $\mathrm{Ng} \mathrm{CSH}, \mathrm{MacDonald} \mathrm{JK}$, Gilbert S, et al. Optimal Approach to Lobectomy for Non-Small Cell Lung 
Cancer: Systemic Review and Meta-Analysis. Innovations 2019;14:90-116.

8. Berry MF, Onaitis MW, Tong BC, et al. Feasibility of hybrid thoracoscopic lobectomy and en-bloc chest wall resection. Eur J Cardiothorac Surg 2012;41:888-92.

9. Rocco G, Fazioli F, Martucci N, et al. Video-assisted thoracic surgery rib resection and reconstruction with titanium plate. Ann Thorac Surg 2011;92:744-5.

10. Nakagiri T, Akashi A, Shigemura N. Thoracoscopic rib resection using a Gigli saw. Ann Thorac Surg 2005;80:755-6.

11. Abicht TO, de Hoyos AL. Chest wall resection and reconstruction: a true thoracoscopic approach. Innovations (Phila) 2011;6:399-402.

12. Widmann MD, Caccavale RJ, Bocage JP, et al. Videoassisted thoracic surgery resection of chest wall en bloc for lung carcinoma. Ann Thorac Surg 2000;70:2138-40.

13. Demmy TL, Nwogu CE, Yendamuri S. Thoracoscopic chest wall resection: what is its role? Ann Thorac Surg 2010;89:S2142-5.

14. Gonzalez-Rivas D, Fernandez R, Fieira E, et al. Singleincision thoracoscopic right upper lobectomy with chest

doi: 10.21037/jovs-20-138

Cite this article as: Brandolini J, Dolci G, Antonacci F, Daddi N, Bertoglio P, Kawamukai K, Bonfanti B, Calabrese F, Fortiparri S, Gasbarrini A, Solli P. Combined approach for NSCLC with chest wall invasion: the role of VATS. J Vis Surg 2021;7:28. wall resection by posterior approach. Innovations (Phila) 2013;8:70-2.

15. Giaccone A, Solli P, Pardolesi A, et al. Video-assisted thoracoscopic surgery en bloc chest wall resection. J Vis Surg 2017;3:73.

16. Caruana EJ, Solli P, Coonar AS. Hybrid video-assisted thoracoscopic surgery lobectomy and en-bloc chest wall resection for non-small cell lung cancer. J Thorac Dis 2016;8:E935-7.

17. Abicht TO, de Hoyos AL. Chest wall resection and reconstruction: a true thoracoscopic approach. Innovations (Phila) 2011;6:399-402.

18. Bayarri CI, de Guevara AC, Martin-Ucar AE. Initial single-port thoracoscopy to reduce surgical trauma during open en bloc chest wall and pulmonary resection for locally invasive cancer. Interact Cardiovasc Thorac Surg 2013;17:32-5.

19. Ferraro P, Cugno S, Liberman $M$ et al. Principles of chest wall resection and reconstruction. Thorac Surg Clin 2010;20:465-73.

20. Sanna S, Brandolini J, Pardolesi A, et al. Materials and techniques in chest wall reconstruction: a review. J Vis Surg 2017;3:95. 